Innovaciones de Negocios 13(26): 239 - 272

(c) 2016 UANL, Impreso en México (ISSN 2007-1191)

Fecha de recepción: 01 de noviembre de 2016. Fecha de aceptación: 02 de diciembre de 2016.

\title{
Impacto de las telecomunicaciones en la gestión de las instituciones de educación superior públicas de México: Un modelo de ecuaciones estructurales (Impact of the telecommunications in the management of the public institutions of higher education of Mexico: A model of structural equations)
}

\author{
Alberto Zambrano Elizondo* \\ María de Jesús Araiza Vázquez* \\ José Fernando Hernández González* \\ Juan Humberto Vela Quintero*
}

\begin{abstract}
The present research had as objective to know information regarding the level of relation of the telecommunications and the user and financial management in the educational institutions of higher level (IES) in Mexico, through a structural equations model. The methodology to know the impact of telecommunications in the management of users and financial management consisted of conducting surveys with 28 questions to a sample of 34 public universities in Mexico. The application of the same was of induced character, and of structured form with questions mostly by a Likert scale. Three hypotheses were formulated that we considered fundamental. To verify, a model of structural equations was designed with the telecommunications service, internal process management, user management and financial management as constructs. With data from the surveys, a cross-sectional database was created and introduced to specialized statistical analysis software called Smart PLS V. 2.0 (M3). The results of the statistical model show that the telecommunications service provided by IHEs in Mexico has a strong relationship with the management of internal processes and this in turn has a very strong relationship with user management and financial management. It is generally concluded that telecommunications that exist in public IES have a strong influence on user management and financial management supported by Internal Process Management.
\end{abstract}

\footnotetext{
- Universidad Autónoma de Nuevo León, Facultad de Contaduría Pública y Administración, Centro de Desarrollo Empresarial y Posgrado, San Nicolás de los Garza, N.L., México. Email: alberto.zambrano@uanl.mx

* Universidad Autónoma de Nuevo León, Facultad de Contaduría Pública y Administración, Centro de Desarrollo Empresarial y Posgrado, San Nicolás de los Garza, N.L., México. Email: maria.araizav@uanl.mx

- Tecnológico de Nuevo Laredo, Nuevo Laredo, Tamaulipas, México, 88275. Email: word110@hotmail.com

+ Tecnológico de Nuevo Laredo, Nuevo Laredo, Tamaulipas, México, 88275. Email: juanhvq@hotmail.com
} 
Keywords: management, process, structural equation model, telecommunications

JEL: $123,031,032$

Resumen. La presente investigación tuvo como objetivo conocer información respecto al nivel de relación de las telecomunicaciones y las gestiones de usuarios y financieras en las instituciones educativas de nivel superior (IES) en México, mediante un modelo de ecuaciones estructurales. La metodología para conocer el impacto de las telecomunicaciones en la gestión de usuarios y gestión financiera, consistió en realizar encuestas con 28 preguntas a una muestra de 34 universidades públicas de México. La aplicación de los mismos fue de carácter inducida, y de forma estructurada con preguntas en su mayoría mediante una escala Likert. Se formularon 3 hipótesis que consideramos fundamentales. Para comprobar, se diseñó un modelo de ecuaciones estructurales con el servicio de las telecomunicaciones, la gestión de procesos internos, gestión de usuarios y la gestión financiera como constructos. Con los datos de las encuestas, se creó una base de datos de corte transversal que se introdujo al software especializado en análisis de datos estadísticos denominado Smart PLS V. 2.0 (M3). Los resultados del modelo estadístico presentan que el servicio de las telecomunicaciones que prestan las IES en México tienen una fuerte relación con la gestión de procesos internos y esta a su vez, tiene una muy fuerte relación con las gestiones de los usuarios y las gestiones financieras. Se concluye de manera general que las telecomunicaciones que existen en las IES públicas tienen una fuerte influencia en las gestiones de usuarios y las gestiones financieras apoyadas con las Gestiones de procesos internos.

Palabras clave: gestión, modelo de ecuaciones estructurales, telecomunicaciones, procesos

\title{
Introducción
}

En un mundo globalizado, donde los constantes cambios en las economías mundiales han orillado a las instituciones públicas y privadas a reformular estrategias enfocadas a su supervivencia, estrategias competitivas que las ayuden a ser más productivas y que aporten utilidades que las impulsen a ser rentables, las instituciones se ven en la necesidad de optimizar los recursos tanto financieros como humanos, materiales y tecnológicos en el corto y mediano plazo.

Para hacer frente a estos cambios, gobiernos y organismos implicados en la educación superior tratan de implementar estrategias para mejorar la eficiencia de las instituciones universitarias que aseguren la correcta utilización de los recursos. Los retos actuales de cualquier gobierno es que

\author{
A. Zambrano, M. J. Araiza, J. F. Hernández \& J. H. Vela
}


las instituciones aumenten su eficiencia y responsabilidad educativa partiendo de la autonomía universitaria (Lou, 2001; Lou, 2007)

Las Tecnologías de Información y Comunicaciones (TIC) han cambiado la naturaleza del trabajo y el tipo de habilidades necesarias en la mayoría de los oficios y profesiones (Kvilon, 2004). Además de las teorías relacionadas a la acción razonada propuestas por Fishbein y Ajzen, citados por García (1997), donde afirman que los seres humanos somos racionales y hacemos uso sistemático de la información que tenemos disponible.

Una elevada optimización en la utilización de recursos tecnológicos, la gestión de procesos internos, de usuarios y financieros entre otros, es algo que siempre desea la dirección de toda organización, porque tiende a traducirse en resultados positivos, generando un alto desarrollo y desempeño organizacional, los cuales al paso del tiempo se ve reflejado en mayores índices de productividad.

Los tiempos de respuesta a los servicios requeridos o prestados, eficiencia, calidad en servicios entre otros, permiten lograr una mayor vinculación entre diversos actores 0 entidades, con el objeto de tomar las decisiones correctas sobre la prevención o solución de las diversas problemáticas que se puedan presentar.

En el proceso permanente del desarrollo del conocimiento, de la acumulación del capital humano y del capital organizativo de las organizaciones, Lastres y Moreno (2002), añaden que la gestión del conocimiento se centra prioritariamente en la tarea de la búsqueda y selección, -entre todos los saberes y habilidades que operan en el entorno de las empresas-, de los que sean claves para el desarrollo de las competencias fundamentales y diferentes que necesita un negocio para competir con éxito, es decir, las que involucran la estrategia relacionada con el denominado . En este entorno tecnológico, la información aumenta su valor económico, circula, se intercambia, se acumula, facilita la mejora de los procesos productivos y estimula la introducción de nuevos bienes y servicios. Por otra parte, las organizaciones en general y las empresas en particular, se enfrentan en este nuevo siglo a uno de los retos más importantes y singular: aprender a dirigir el conjunto del saber existente, con el fin de crear valor en esta nueva sociedad del conocimiento y generar ventajas competitivas sostenibles, que les faciliten lograr la excelencia organizativa y la calidad de su gestión. 
La presente investigación tiene como objetivo analizar y conocer el impacto de las telecomunicaciones (TELE) en la Gestión de Procesos internos (GPI) y esta a su vez, en el impacto en la Gestión de Usuarios (GU) y la Gestión Financieras (GF), mediante un modelo de ecuaciones estructurales y su importancia fue contribuir al conocimiento de los factores que definen una efectividad frente a la utilización de las Telecomunicaciones en las IES en México, dependiendo de las percepciones económicas, sociales, laborales, de seguridad, y oportunidades de progreso de cada institución.

Por lo tanto, nuestra pregunta de investigación fue: ¿Cuál los indicadores que definen una efectividad frente a la utilización de las telecomunicaciones en la Gestión de las IES públicas?

\section{Marco teórico}

La universidad es considerada como el espacio social por excelencia donde confluyen las ideas, las reflexiones y el intelecto, que contribuyen a transformar el ser y el quehacer de la sociedad.

La literatura ofrece diversidad de indicadores organizados en base a diferentes criterios: unos evalúan solamente la docencia, otros la investigación, otros la transferencia de los resultados de investigación o la evaluación conjunta de todas ellas, aportando diferentes inputs u outputs

\section{El impacto de las telecomunicaciones}

En la actualidad, las telecomunicaciones constituyen uno de los sectores más importantes para cualquier país ya que contribuye al desarrollo económico, social, y mejora la calidad de vida de la población (UIT, 2000). "A nivel mundial existe una gran disparidad significativa entre regiones que gozan de los beneficios de las comunicaciones y aquellas donde el acceso a este tipo de servicios es limitado," (Serrano, 2000)

Es indudable que las telecomunicaciones tienen presencia mundial, gracias al proceso de globalización. También es evidente que el proceso de globalización en todas sus dimensiones, pero en especial la económica, se ha beneficiado de la apertura de las telecomunicaciones a nivel mundial. Bien 
sabemos, solo se prospera con una tecnología reconociendo su accidente especifico, su negatividad inherente. Las TIC constituyen el vehículo para un cierto tipo de accidente que ya no es local, es un accidente que involucra inmediatamente a la totalidad del mundo. El accidente de Internet, o el accidente de otras tecnologías de la misma especie, es también la aparición de un accidente total, por no decir integral. (Recordemos aquí el famoso problema del año 2000, Y2K, y los grandes costos, para solucionarlo). Gracias a las telecomunicaciones, somos una sola aldea, podemos estar virtualmente en cualquier parte del globo.

\section{Las TIC y la gestión}

El uso y gestión de las telecomunicaciones ha traído como consecuencia: el cambio en la estructura de los sectores productivos, que se basan cada vez más en redes de colaboración de producción, gestión e intercambio de información, la elevación del nivel de estudios de una parte significativa de la población mundial y, por lo tanto, de su capacidad de manejar información, la convergencia informática-comunicaciones se hace total en los usos actuales de Internet, las organizaciones empiezan a gestionar información y conocimientos, y no sólo datos como en los inicios, utilizando las TIC, la difusión de las TIC en el uso social diario no cesa de aumentar, tanto en los hogares como en las organizaciones.

Debemos considerar que la organización con el apoyo de las TIC tiene múltiples ventajas y oportunidades, tanto en productividad como en inclusión, logrando agregar valor al negocio. Puede sumar concentración de información en corto tiempo, nuevos modelos económicos, reputación online y transparencia de las misma, entre otros.

De acuerdo a Macau (2004), la automatización del proceso administrativo y burocrático es una función obligada en la organización moderna. Desde el punto de vista directivo se gestiona con criterios clásicos. Las TIC permiten plantearse, de manera económicamente realista, actuaciones transversales e integradoras en los circuitos administrativos de una organización.

Los servicios de información, como parte esencial de la infraestructura para la gestión del conocimiento, suministran información, impulsan la generación del conocimiento para la búsqueda de soluciones a los problemas 
que enfrentan las organizaciones, analizan su impacto sobre los resultados de las empresas e influyen en el comportamiento de los individuos ante la información. La gestión de la información se vincula con la generación y la aplicación de estrategias, el establecimiento de políticas, así como con el desarrollo de una cultura organizacional y social dirigida al uso racional, efectivo y eficiente de la información en función de los objetivos y metas de las compañías en materia de desempeño y de calidad. (Aja, 2002)

\section{Las IES hoy en día}

El surgimiento de la sociedad del conocimiento, el fenómeno de la mundialización de los servicios, la revolución científico-técnica y el progresivo interés por la economía del bienestar promueve, en los países con economías competitivas, la aparición de un nuevo modelo de universidad que adopta de forma creciente la llamada «tercera misión» en el ámbito de los ejes vertebradores como el emprendimiento, la innovación y el compromiso social (Bricall, 2000; Commission of the European Communities, 2006; Bueno, 2007; Gulbrandsen y Slipersaeter, 2007).

En este contexto sociopolítico, se configura un nuevo esquema de relaciones entre las universidades, los centros de investigación, la administración pública y las empresas (OCDE, 1999; Bricall, 2000), surgiendo la denominada "universidad emprendedora» (Clark, 1997, 1998; Davies, 2001).

Según Palomares (2008), el sistema universitario está experimentando profundos cambios que están influyendo directamente en el desarrollo socioeconómico de un país. Según estudios de la OCDE (1999), las universidades están aplicando nuevos roles y tendencias que tienen serias implicaciones en su estructura.

Para hacer frente a estos cambios, gobiernos y organismos implicados en la educación superior tratan de implementar estrategias para mejorar la eficiencia de las instituciones universitarias que aseguren la correcta utilización de los recursos. Los retos actuales de cualquier gobierno es que las instituciones aumenten su eficiencia y responsabilidad educativa partiendo de la autonomía universitaria (Lou, 2001; Lou, 2007) 
Keller (1999), Villarreal (1999), Pla \& Villarreal (2001) y García et al. (2006) mencionan que se ha llegado el momento en que las universidades deben ser evaluadas. La evaluación de la educación superior es un proceso complejo que debe realizarse de acuerdo con unos estándares válidos y fiables (Díaz, 1999). Por esta razón, en las últimas décadas están surgiendo numerosos estudios acerca de la evaluación de las universidades mediante la utilización de sistemas de indicadores (Consejo de Universidades, 1999).

\section{Materiales y métodos}

En este apartado haremos referencia al cuestionario utilizado y a los métodos de análisis utilizados para cumplir con nuestros objetivos.

Instrumento de medición

Para esta investigación se elaboró y se aplicó un cuestionario que contempla cuatro secciones centrales sobre las cuales recae la preocupación por conocer el impacto de las telecomunicaciones en las gestiones de los usuarios y las gestiones financieras que realizan las IES.:

- Datos generales de la institución

- Servicios de telecomunicaciones que se ofrecen en las IES

- Gestión de Procesos Internos en las IES

- Gestión de Usuarios y Financieras que se realizan en una IES

El primer bloque denominado datos generales, incluye indicadores sociodemográficos que permiten conocer número de estudiantes, personal aulas, presupuesto anual, número de equipos, tipo de equipos, utilización de equipos por tipo d usuario, entre otros indicadores.

El segundo bloque referente a esta investigación contempla 11 indicadores en torno a los servicios de telecomunicaciones, como servicios de ancho de banda, acceso a la red Nacional de Impulso a la Banda Ancha (INBA), servicio de acceso a internet, servicios de video vigilancia, telefonía, red de datos entre otras. Estos indicadores nos ayudarán a cumplir con el objetivo final del diagnóstico en relación a conocer el nivel de las variables antes mencionadas que puedan servir como base para la formulación de 
estrategias que puedan eficientar la utilización de las telecomunicaciones en las IES de México.

El tercer bloque contempla 8 indicadores relacionados a las gestiones de los procesos internos en las IES como: reducciones de tiempo en la ejecución de tareas administrativas, cumplimiento de leyes externas, regulaciones y contratos, eficiencia en los procesos institucionales entre otros

El tercer y cuarto bloque está relacionado a la gestión de usuarios y la gestión financiera respectivamente que se realiza en las IES; contemplan en conjunto 9 indicadores como la calidad y cantidad de investigaciones realizadas, la calidad de los servicios académicos, y administrativos que reciben los usuarios, la eficiencia en los costos, la planificación y ejecución del presupuesto, entre otros indicadores no menos importantes

Por último, es importante precisar que la mayor parte de la información obtenida para este diagnóstico es de carácter inédito que puede ser de utilidad en la planificación de programas y políticas institucionales orientadas a propiciar mejores oportunidades y condiciones en las IES de México. Puesto que, en este estudio, nos enfocamos directamente al análisis e interpretación de los datos obtenidos los bloques anteriores de dicha encuesta.

Métodos

La encuesta es aplicada a través de un cuestionario, este método normalmente es utilizado para obtener información relativa a las características predominantes de una población mediante la aplicación de procesos de interrogación y registro de datos, se pude conseguir principalmente información demográfica, opiniones y conocimientos de los sujetos respecto a un asunto, situación, tema o personas. Es necesario aclarar que la encuesta es una actividad consiente y planeada para indagar y obtener hechos, conocimientos, opiniones, juicios y motivaciones. Esto se logra a través de la preguntas orales o escritas que ordinariamente generan reflexión a un conjunto de preguntas previamente diseñadas que se aplican en forma masiva; con ello se concentran datos relativos a sucesos ya ocurridos, es decir, se recoge información de situaciones ya pasadas. (Córdoba, 2002 p.20-21). 
Debido a las implicaciones técnicas que representaba la realización de entrevistas al personal en general de las IES de México, se realizaron encuestas a las universidades más importantes de México con reconocimiento nacional e internacional.

Definición de hipótesis

La formulación de la hipótesis de investigación está basada en el procedimiento recomendado por Kerlinger (2002), donde se parte de la definición de una hipótesis sustantiva en la cual se expresa una información conjetural de la relación entre dos o más variables.

El desarrollo de esta investigación nos llevó a crear y comprobar algunas hipótesis que consideramos importantes en tema de las telecomunicaciones, servicios y gestión que se llevan a cabo en las universidades públicas de México., las cuales se describen a continuación: En la figura 1, se plantean y expresan las hipótesis en forma conceptual.

Figura 1. Mapa conceptual de hipótesis planteadas

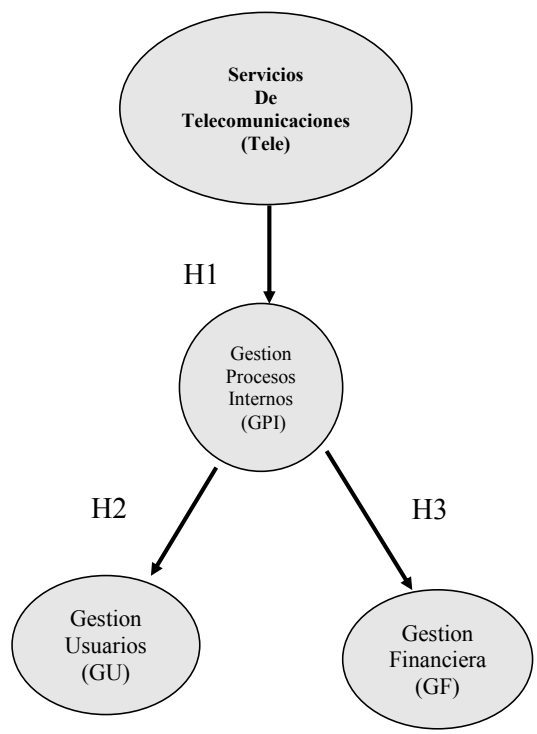

Fuente: Elaborada por los autores. 
En muchas instituciones se tiene la percepción que las telecomunicaciones mejoran la gestión de los procesos internos de las instituciones de educación superior, por lo tanto, se generó la siguiente hipótesis.

$H_{1}$ : A mejor infraestructura en telecomunicaciones, no mejora la gestión de procesos internos.

Las IES que muestran mejoras en sus procesos internos, mejoran de la gestión realizada por sus usuarios.

$\mathrm{H}_{2}$ : A mejor gestión en los procesos internos, no se refleja en una mejora de la gestión realizada por los de usuarios.

Las IES muestran que, a una mejora en los procesos internos, mejor es la gestión Financiera.

$H_{3}$ : A mejor gestión en los procesos internos, no es mejor la gestión financiera.

Una mejor gestión en los procesos internos, no se refleja en una mejor gestión financiera

\section{Muestreo}

Al elegir una muestra se espera conseguir que sus propiedades sean extrapolables a la población (Garza, Lerma y Terriquez., 2014). Esto nos permite ahorro significativo de costos y recursos, y también es como si se realizara un estudio completo a toda la población.

La ANUIES en su anuario de estadísticos de educación superior muestra todas las universidades públicas de México; se tomó como referencia para definir el grupo de muestras en esta investigación. El tipo de muestreo utilizado en esta encuesta es de carácter aleatorio. Es un tipo de muestreo utilizado primero porque la población es finita y homogénea y es por eso que se inclinó por este tipo de selección. Es aleatorio porque todas las unidades de muestreo tienen una probabilidad conocida y distinta de cero de ser seleccionadas (ver Tabla 1). 
Tabla 1. I.E.S. públicas de México

\begin{tabular}{|c|c|}
\hline No. & Universidad \\
\hline 1 & Universidad Autónoma de Ciudad Juárez \\
\hline 2 & Universidad de Colima \\
\hline 3 & Universidad Autónoma de Yucatán \\
\hline 4 & Instituto Tecnológico de Sonora \\
\hline 5 & Universidad Autónoma de Aguascalientes \\
\hline 6 & Universidad Autónoma del Carmen \\
\hline 7 & Universidad Autónoma de Nuevo León \\
\hline 8 & Universidad Autónoma de Querétaro \\
\hline 9 & Universidad Autónoma de Tlaxcala \\
\hline 10 & Universidad Autónoma Benito Juárez de Oaxaca \\
\hline 11 & Universidad de Guadalajara \\
\hline 12 & Universidad Autónoma Metropolitana \\
\hline 13 & Universidad Autónoma de San Luis Potosí \\
\hline 14 & Universidad Veracruzana \\
\hline 15 & Universidad Autónoma de Campeche \\
\hline 16 & Universidad Autónoma de Tamaulipas \\
\hline 17 & Universidad Juárez del Estado De Durango \\
\hline 18 & Universidad Autónoma de Coahuila \\
\hline 19 & Universidad de Guanajuato \\
\hline 20 & Universidad Autónoma del Estado De México \\
\hline 21 & Universidad Autónoma de Guerrero \\
\hline 22 & Universidad Autónoma Agraria Antonio Narro \\
\hline 23 & Universidad Autónoma del Estado De Hidalgo \\
\hline 24 & Universidad Nacional Autónoma de México \\
\hline 25 & Universidad de Quintana Roo \\
\hline 26 & Universidad Autónoma de Chihuahua \\
\hline 27 & Universidad Autónoma del Estado De Morelos \\
\hline 28 & Universidad Juárez Autónoma de Tabasco \\
\hline 29 & Universidad Autónoma de Baja California Sur \\
\hline 30 & Universidad Pedagógica Nacional \\
\hline 31 & Universidad de Sonora \\
\hline 32 & Universidad Autónoma de Chiapas \\
\hline 33 & Universidad Autónoma del Estado de Baja California \\
\hline 34 & Benemérita Universidad Autónoma de Puebla \\
\hline
\end{tabular}


Bajo estos criterios, el tamaño final de la muestra fue de 34 Universidades públicas distribuidas en todo el país. El esquema de muestreo contempló un nivel de confianza del $95 \%$, un error relativo máximo esperado de $3.0 \%$, una tasa anticipada de no respuesta del $30 \%$ y un efecto de diseño de 1, para propósitos de estimación de las variables dicotómicas. Debido a que no se contaba con antecedentes previos, se fijó la proporción al $50 \%$.

En cuanto al tamaño de muestra, atendiendo a que también se manejan ecuaciones estructurales con SmartPLS, dado que el número de constructos es de 4, el tamaño de muestra mínimo es de 33 encuestas, que es sobrepasado por el tamaño de muestra que se realizó para los propósitos de estimación que se mencionan al principio de este párrafo (Rositas, 2014).

La aplicación de la encuesta fue de forma estructurada con preguntas que se evalúan en su mayoría mediante una escala Likert. Con los datos de las encuestas, se creó una base de datos de corte transversal que se introdujo al software especializado en análisis de datos estadísticos denominado SmartPLS V. 2.0 (M3).

\section{Resultados}

Los primeros pasos hacia la sociedad de la información del conocimiento de México se dieron formalmente en el Plan Nacional de Desarrollo 1995 - 2000 donde se introduce un programa de desarrollo informático. Luego para 2001 se crea la ciberestrategia nacional denominada "Sistema Nacional e-México" con el fin de aprovechar el potencial de las TIC para apoyar los grandes objetivos nacionales entre los que se encontraba la educación y otros servicios a la comunidad cuya finalidad fue fomentar el desarrollo de la ciencia y la tecnología. Es aquí precisamente donde México establece el compromiso ante el mundo en materia de uso y aplicación de las TIC al suscribir la "Declaración de Principios" durante la 1a. Fase de la Cumbre Mundial de la Sociedad de la Información en Ginebra Suiza. (C. M. S. I., 2003). Esto provoco a que actores gubernamentales y particulares se inscribieran a coadyuvar en esta tarea. Las Universidades no pudieron ser ajenas a esta petición sabiendo que uno de los 6 objetivos que fueron establecidos fue TIC en la educación. De ahí el interés de conocer como está 
impactando las telecomunicaciones a los servicios ofrecidos por las IES a sus actores.

Datos demográficos

Dentro de las IES públicas consideradas para este estudio podemos observar datos específicos con los que cada una de ellas trabajan. Muchos de ellos basados en tamaño de población y oferta educativa (Ver Figura 2). Para poner en contexto al lector, de la misma manera mostramos la cantidad de estudiantes, docentes e investigadores que la conforman al momento de hacer el estudio ver Figuras 3, 4 y 5, todo ello para darnos una idea de las dimensiones de cada una.

Es importante destacar en este estudio, los elementos que determinan 0 intervienen en la gestión de los recursos versus las cantidades de los usuarios atendidos, así como la infraestructura tecnológica que se gesta para dar servicios a sus usuarios dentro de las instituciones educativas, pueden apreciarse dichas características en las gráficas desde la Figura 2 hasta la Figura 10.

Fig. 2. Presupuestos de las IES públicas de México

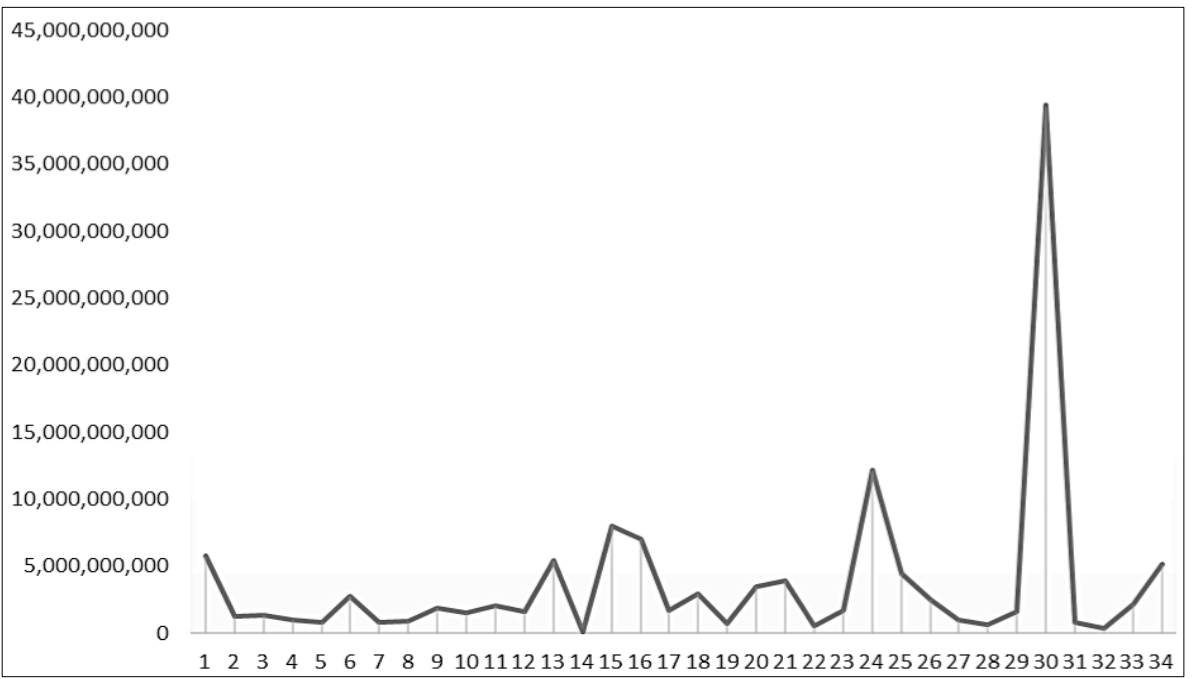

Fuente: Elaboración hecha por los autores. 
Figura 3. Estudiantes por semestre por IES pública de México al momento del estudio

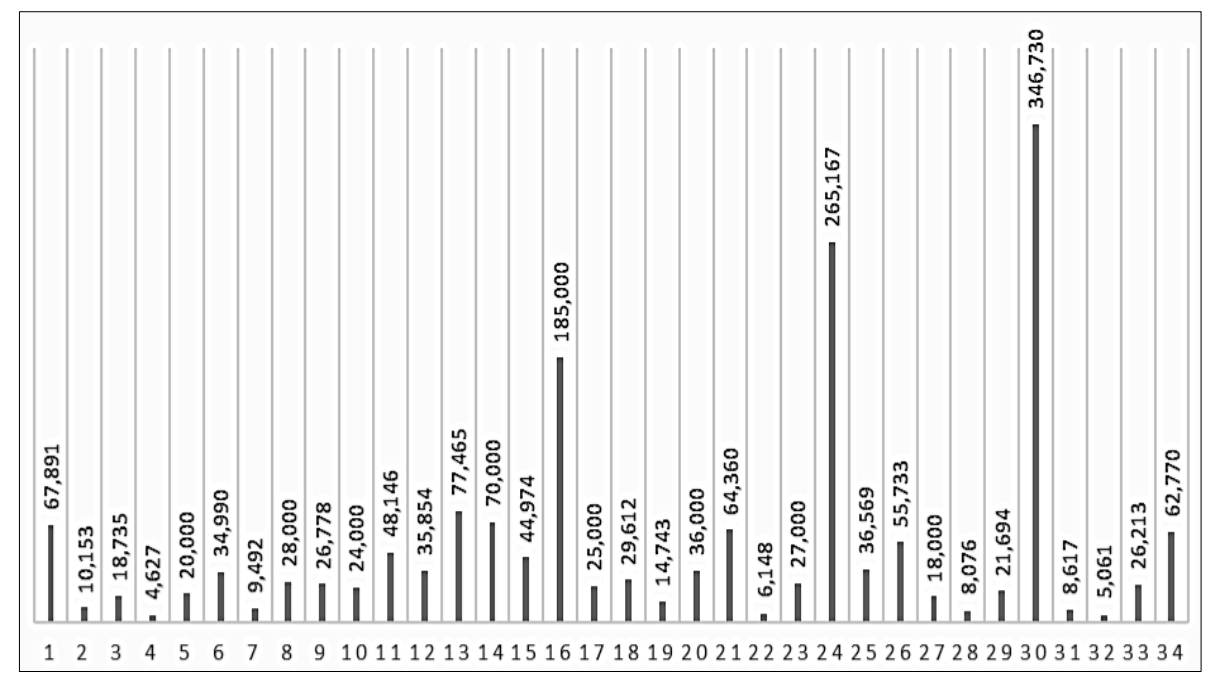

Fuente: Elaboración hecha por los autores.

Figura 4. Docentes por semestre por IES pública de México al momento del estudio

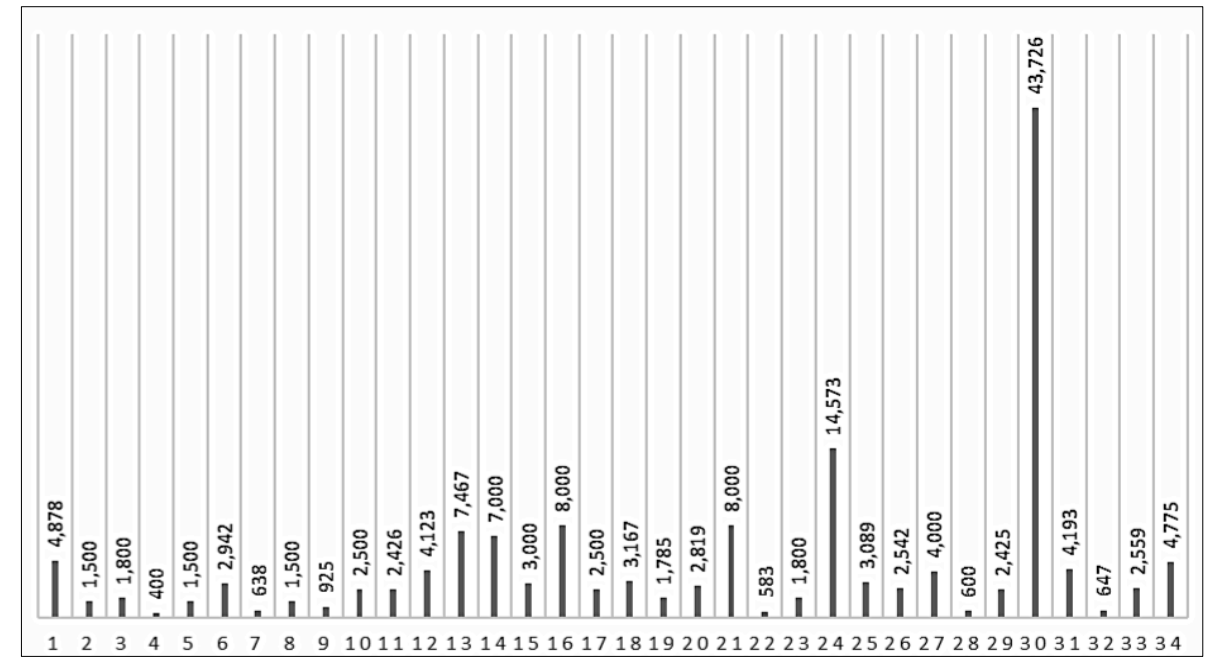

Fuente: Elaboración propia.

A. Zambrano, M. J. Araiza, J. F. Hernández \& J. H. Vela 
Figura 5. Investigadores, por semestre por IES pública de México al momento del estudio

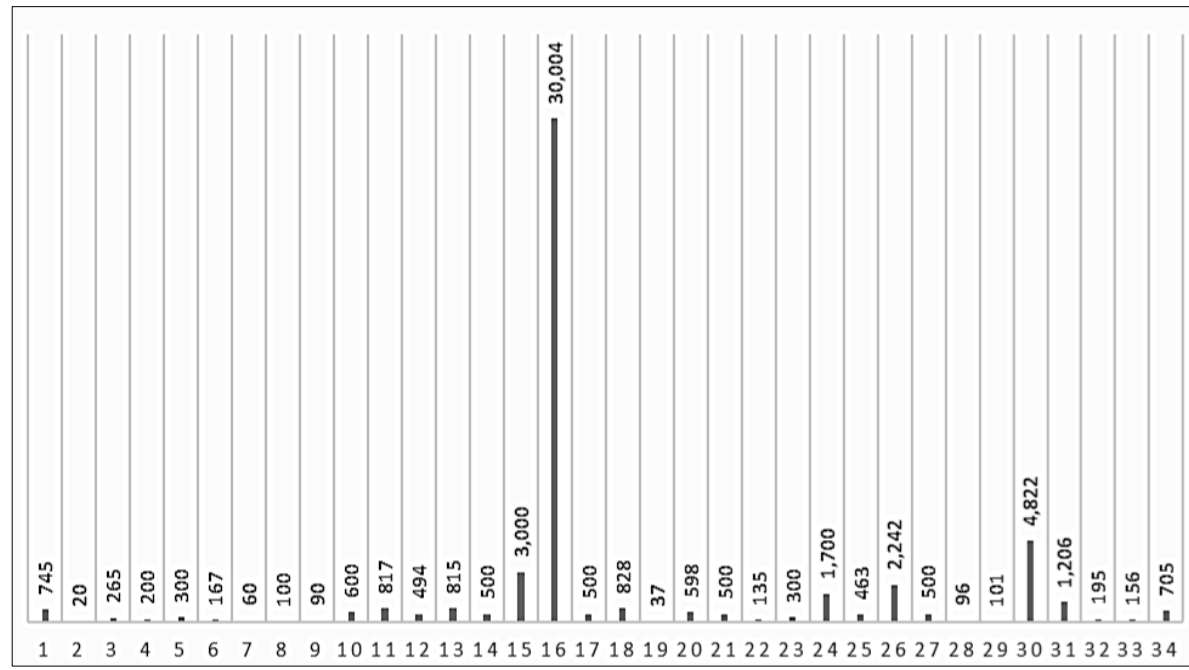

Fuente: Elaboración propia.

Figura 6. Personal de tecnologías de información por IES pública de México al momento del estudio

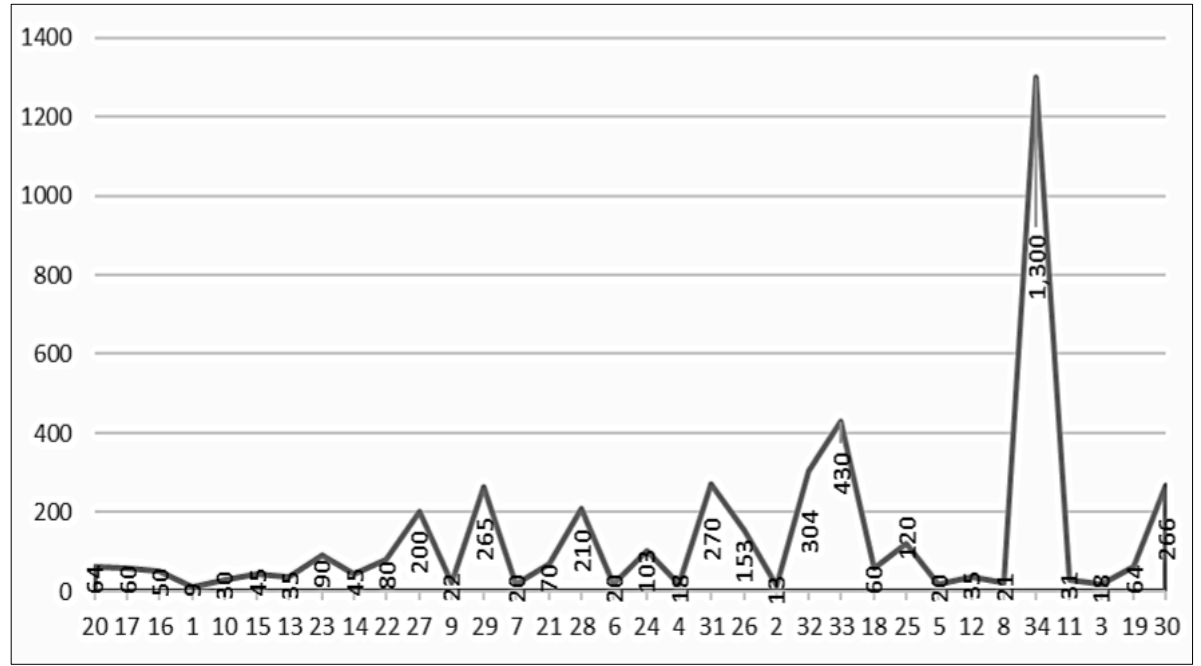

Fuente: Elaboración propia. 
Figura 7. Servidores de cómputo por IES pública de México al momento del estudio

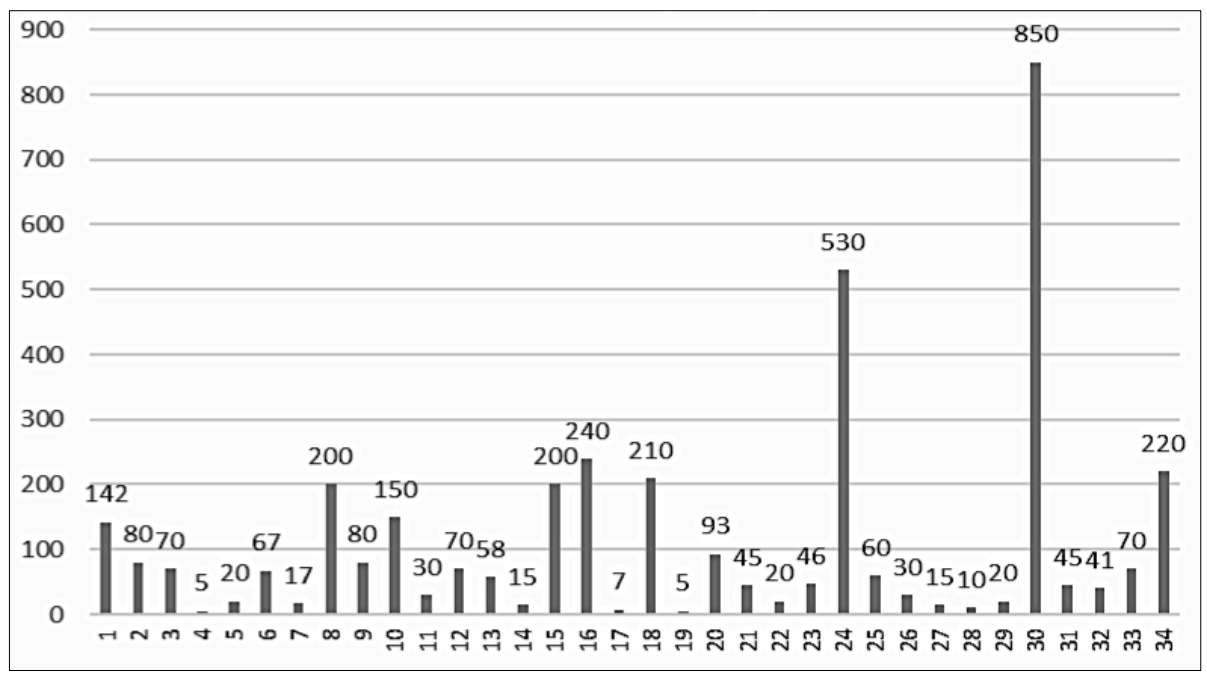

Fuente: Elaboración propia.

Figura 8. PC's dedicadas a la administración de la IES pública de México al momento del estudio

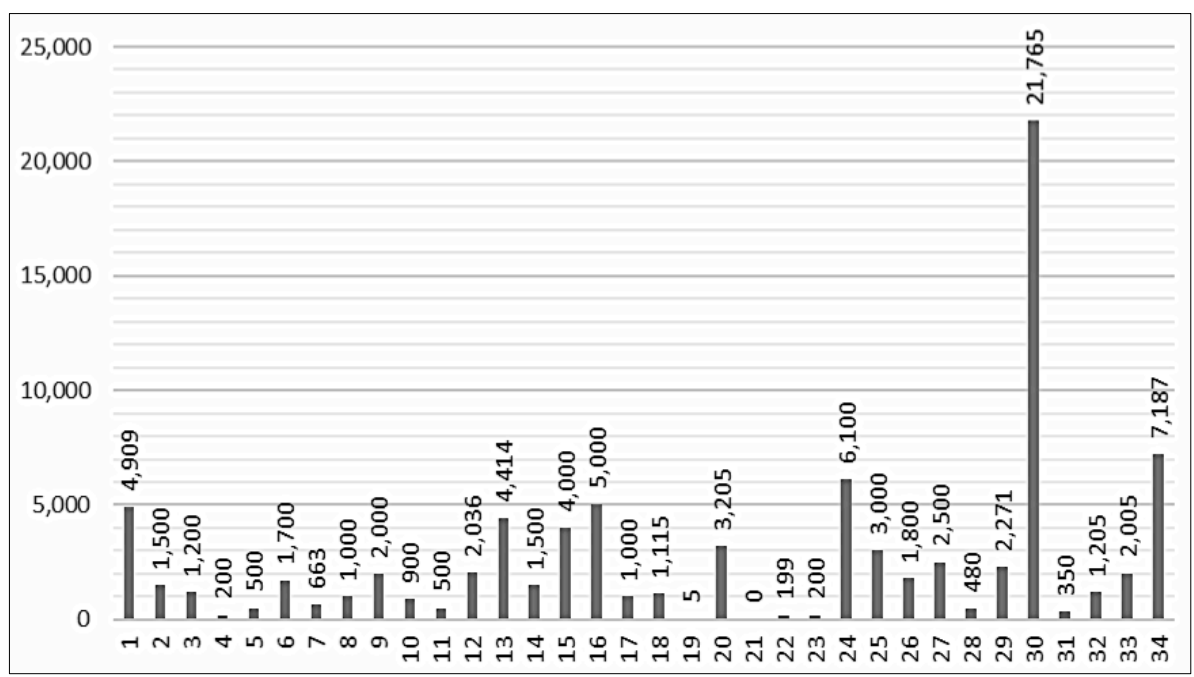

Fuente: Elaboración propia. 
Figura. 9. Estudiantes conectados a la red inalámbrica por mes de la IES pública de México al momento del estudio

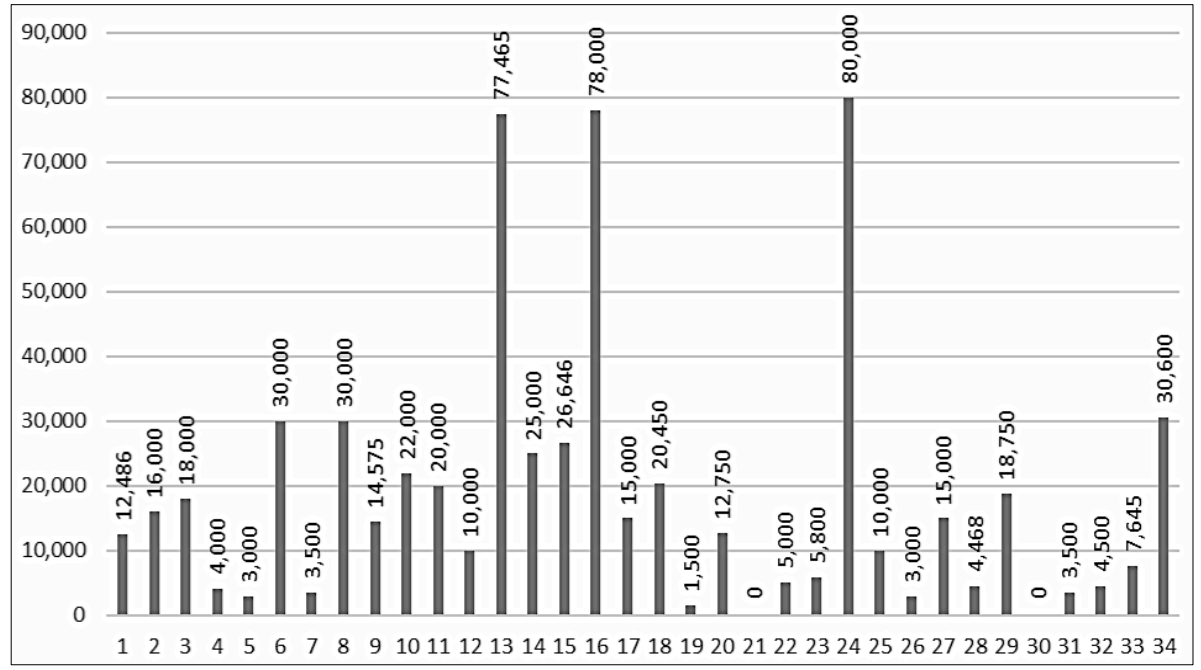

Fuente: Elaboración propia.

Figura 10. Docentes conectados a la red inalámbrica por mes de la IES pública de México al momento del estudio

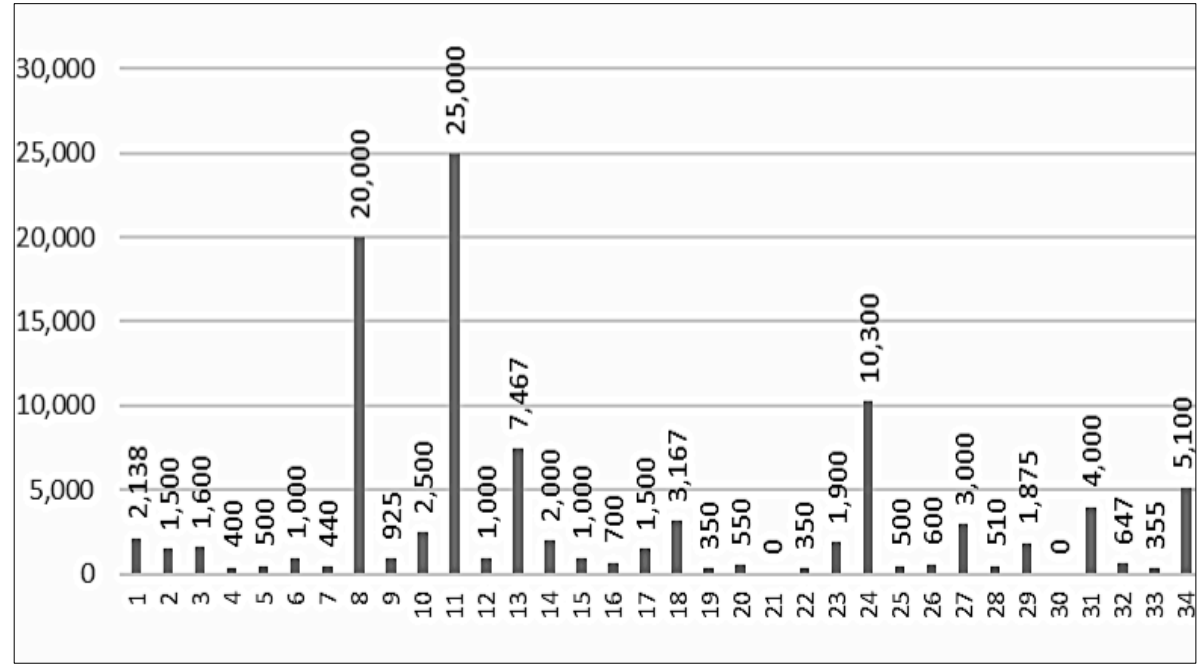

Fuente: Elaboración propia. 
Modelo de ecuación estructural de variables latentes

A continuación, se muestra un modelo inicial que contiene los constructos Servicios de Telecomunicaciones, Procesos Internos, Gestión Usuarios y gestión Financiera (TPUF) que se definieron previamente en esta investigación para las Instituciones de Educación Superior Publicas (IES).

Figura 11. Modelo de ecuación estructural TPUF

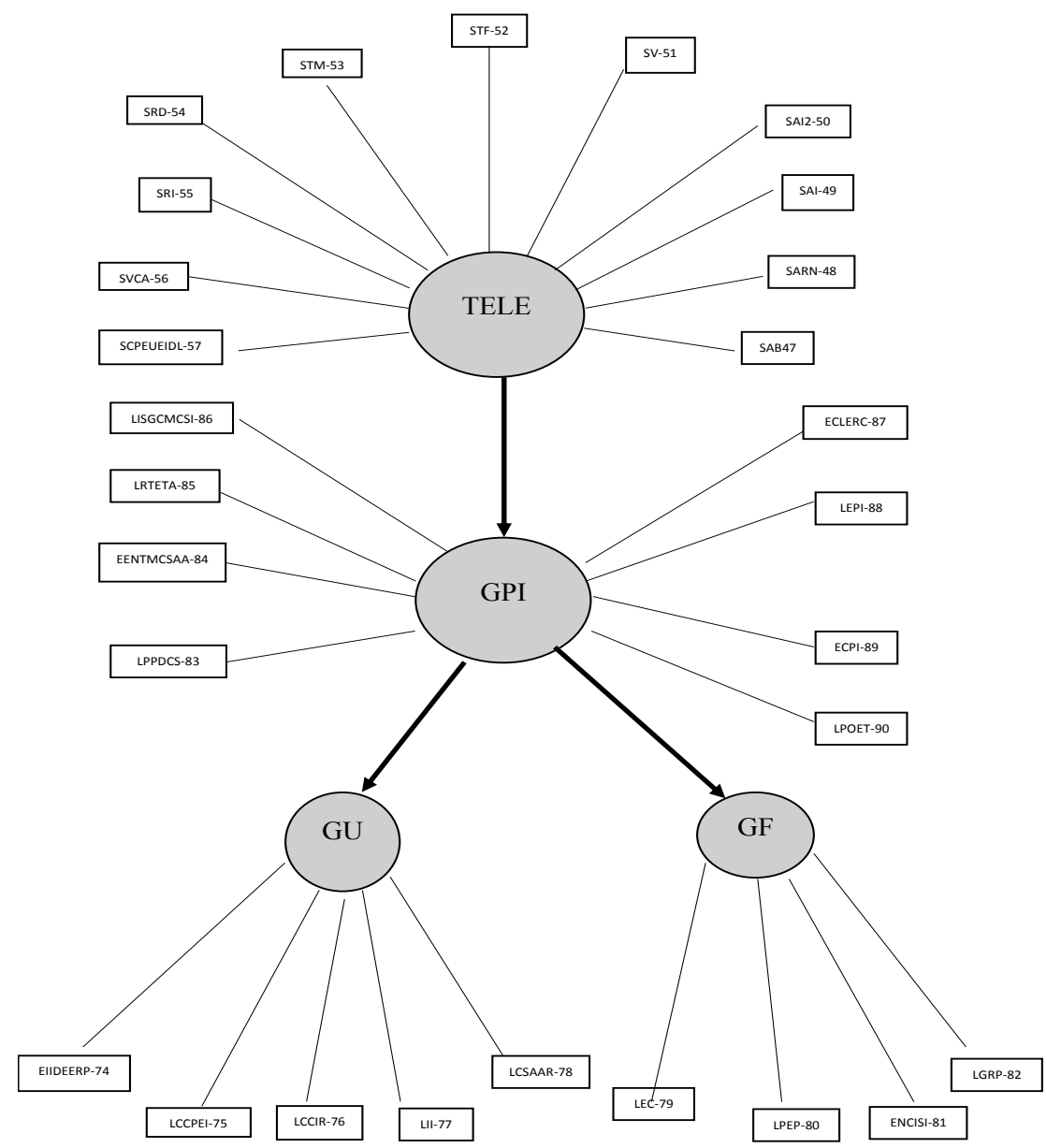

Fuente: Elaborada por los autores utilizando el software SmartPLS 
En las Tablas 2, 3, 4 y 5 se describen las escalas de medición de los constructos evaluados en esta investigación.

Tabla 2. Constructos de servicios y telecomunicaciones y sus ítems

\begin{tabular}{ll}
\hline \multicolumn{1}{c}{ Ítem } & \multicolumn{1}{c}{ Descripción } \\
\hline SAB_54 & Servicio de Ancho de Banda \\
SAR_55 & Servicio de Acceso a la Red NIBA (Nacional de Impulso a la Banda Ancha) \\
SAI_56 & Servicio de Acceso a Internet (Comercial) \\
SAI2_57 & Servicio de Acceso a Internet2 (CUDI) \\
SVI_58 & Servicio de Videoconferencia \\
STF_59 & Servicio de Telefonía Fija \\
STM_60 & Servicio de Telefonía Móvil \\
SRD_61 & Servicio de Red de Datos(Wired) \\
SRI_62 & Servicio de Red Inalámbrica(Wirless) \\
SVyCA_63 & Servicio de Video vigilancia y Control de Accesos (edificios y áreas públicas) \\
SPEUPS_64 & Servicio de climas, Plantas de Emergencia, UPS, Extinciones Incendios y \\
& Detección de Líquidos)
\end{tabular}

Fuente: Elaborada por los autores.

Tabla 3. Constructos gestión de procesos internos y sus ítems

\begin{tabular}{ll}
\hline \multicolumn{1}{c}{ Ítem } & \multicolumn{1}{c}{ Descripción } \\
\hline PPDSC_91 & La Promoción, preservación y desarrollo de la cultura hacia la sociedad \\
RTE_92 & $\begin{array}{l}\text { El empleo de nuevas tecnologías para mejorar la calidad de los servicios } \\
\text { administrativos y académicos } \\
\text { La reducción de tiempo de ejecución de tareas administrativas (reducir tiempo } \\
\text { de espera del usuario) } \\
\text { ENT_93 }\end{array}$ \\
ISGC_94 & $\begin{array}{l}\text { calidad de los servicios institucionales } \\
\text { El cumplimiento de las leyes externas, regulaciones y contratos }\end{array}$ \\
CLERC_95 & $\begin{array}{l}\text { La eficiencia de los procesos institucionales } \\
\text { EPI_96 }\end{array}$ \\
CPI_97 & El cumplimiento de las políticas institucionales (normatividad interna) \\
PO_98 & La productividad operativa de los equipos de trabajo
\end{tabular}

Fuente: Elaborada por los autores. 
Tabla 4. Constructos gestión financiera y sus ítems

\begin{tabular}{|c|c|}
\hline İtem & Descripción \\
\hline EC_87 & La eficiencia en costos (reducir los costos) \\
\hline PEP_88 & La planificación y Ejecución del Presupuesto \\
\hline $\mathrm{NCl} \_89$ & El nivel de Captación de Ingresos por los servicios institucionales \\
\hline GRP_90 & $\begin{array}{l}\text { La generación de Recursos Propios (servicios externos por consultoría o } \\
\text { investigación) }\end{array}$ \\
\hline
\end{tabular}

Fuente: Elaborada por los autores.

Tabla 5. Constructos gestión usuarios y sus ítems

\begin{tabular}{ll}
\hline \multicolumn{1}{c}{ Ítem } & \multicolumn{1}{c}{ Descripción } \\
\hline EIIDEEPR-74 & $\begin{array}{l}\text { El impacto de su institución en el desarrollo económico del Estado, Región y } \\
\text { País. }\end{array}$ \\
LCCPEI-75 & La calidad y cantidad de los Profesionistas egresados de su institución. \\
LCCIR-76 & La calidad y la cantidad de las investigaciones realizadas. \\
LII-77 & La imagen de su Institución. \\
LCSAAR-78 & La calidad de los servicios académicos y administrativos que reciben. \\
\hline
\end{tabular}

Fuente: Elaborada por los autores.

A continuación, presentamos la especificación, estimación y evaluación del modelo con la metodología propuesta.

A) Especificación del modelo

En la Figura 12 se presenta el modelo TPUF mediante ecuaciones estructurales definidas en esta investigación, donde se contemplan los constructos creados y sus indicadores más significativos.

\author{
A. Zambrano, M. J. Araiza, J. F. Hernández \& J. H. Vela
}


Figura 12. Modelo de ecuación estructural TPUF

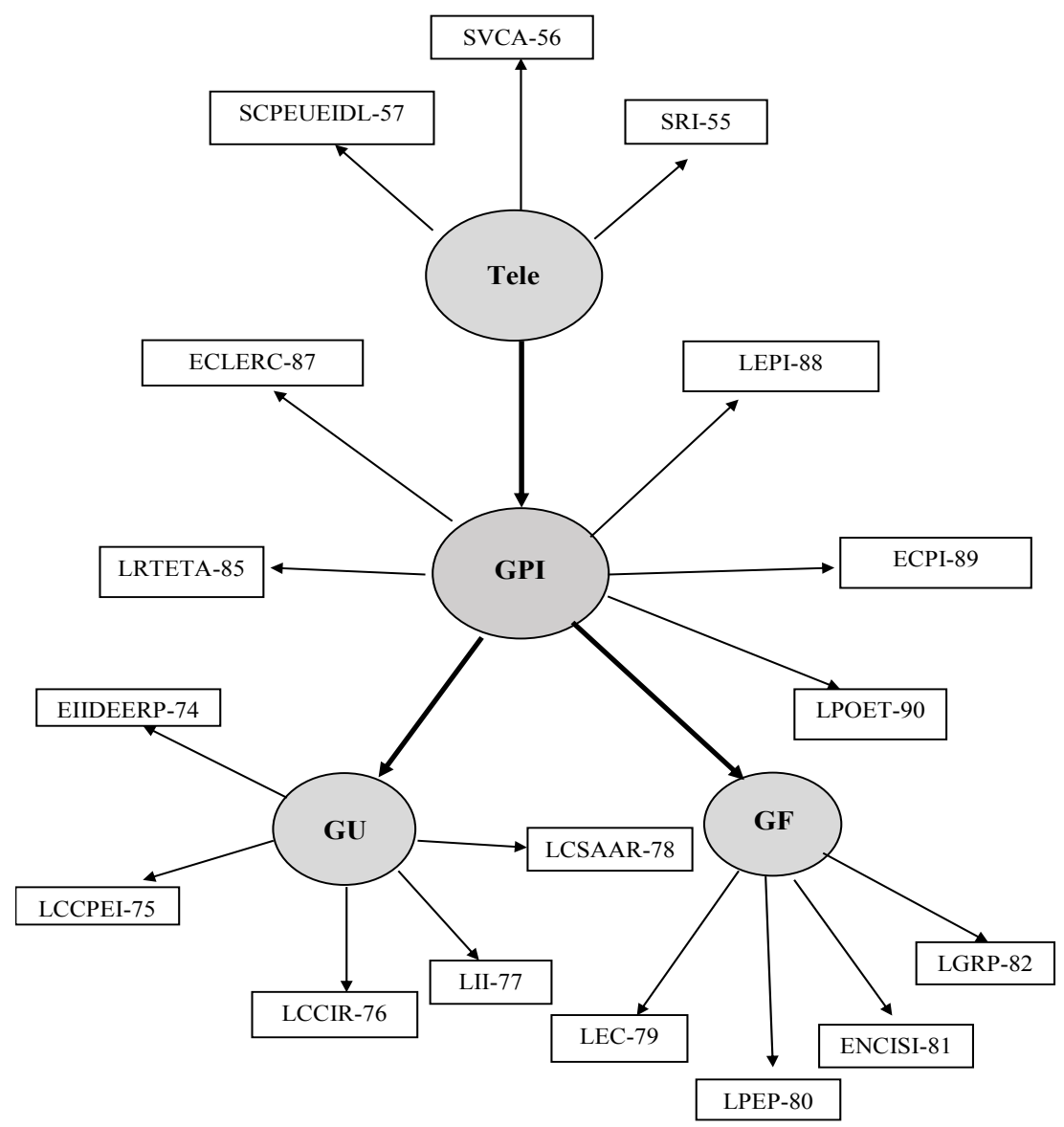

Fuente: Elaborada por los autores utilizando el software SmartPLS.

En la Tabla 6 se muestra la descripción de los constructos y sus variables significativas en el modelo de impacto de las Telecomunicaciones, Gestión de Procesos Internos, Gestión de Usuarios y la Gestión Financiera en las IES. 
Tabla 6. Descripción constructos y sus items

\begin{tabular}{|c|c|}
\hline Clave & Descripción \\
\hline \multicolumn{2}{|r|}{ Constructo Servicios de Telecomunicaciones. } \\
\hline SRI-55 & Servicio de Red Inalámbrica. \\
\hline SVCA-56 & Servicio de video vigilancia y Control de Accesos. \\
\hline CCPUEIDL-57 & $\begin{array}{l}\text { Servicio de Climas, Plantas de Emergencia, UPS, Extinción de Incendios y Detección } \\
\text { de Líquidos. }\end{array}$ \\
\hline \multicolumn{2}{|r|}{ Constructo Gestión de Procesos Internos. } \\
\hline LRTETA-85 & $\begin{array}{l}\text { La Reducción del tiempo de ejecución de tareas administrativas (Reducir tiempos de } \\
\text { espera de los usuarios). }\end{array}$ \\
\hline ECLERC-87 & El cumplimiento con las leyes externas, regulaciones y contratos. \\
\hline LEPI-88 & La eficiencia de los procesos institucionales. \\
\hline ECPI-89 & El cumplimiento de las políticas institucionales. \\
\hline LPOET-90 & La productividad operativa de los equipos de trabajo. \\
\hline \multicolumn{2}{|r|}{ Constructo Gestión Usuarios. } \\
\hline EIIDEEPR-74 & El impacto de su institución en el desarrollo económico del Estado, Región y País. \\
\hline LCCPEI-75 & La calidad y cantidad de los Profesionistas egresados de su institución. \\
\hline LCCIR-76 & La calidad y la cantidad de las investigaciones realizadas. \\
\hline LII-77 & La imagen de su Institución. \\
\hline LCSAAR-78 & La calidad de los servicios académicos y administrativos que reciben. \\
\hline \multicolumn{2}{|r|}{ Constructo Gestión Financiera. } \\
\hline LEC-79 & La eficiencia en los costos (Reducir los costos). \\
\hline LPEP-80 & La planificación y ejecución del presupuesto. \\
\hline ENCISI-81 & El nivel de captación de ingresos por los servicios institucionales. \\
\hline LGRP-82 & $\begin{array}{l}\text { La generación de recursos propios (servicios externos por consultoría o } \\
\text { investigación). }\end{array}$ \\
\hline
\end{tabular}

Fuente: Elaborada por los autores.

B) Estimación del modelo mediante Smart PLS

A continuación, presentamos el modelo de impacto con sus coeficientes de regresión, así como el modelo que muestra la significancia de estos coeficientes mediante el método de bootstrapping utilizado por el SmartPLS.

\author{
A. Zambrano, M. J. Araiza, J. F. Hernández \& J. H. Vela
}


Figura 13. Algoritmo PLS para los 4 constructos del modelo TPUF

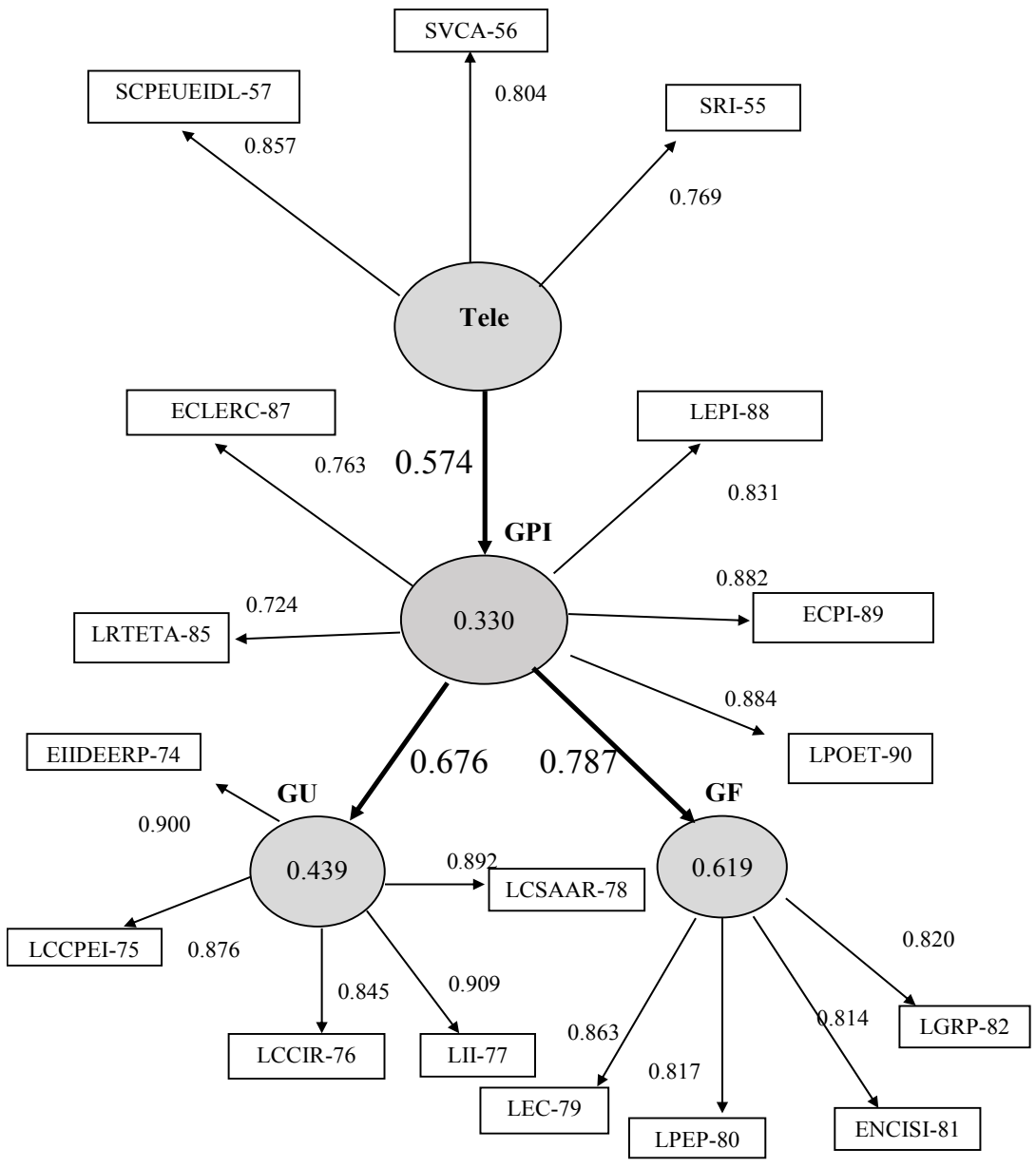

Fuente: Elaborada por el autor utilizando el software SmartPLS

C) Ejecución Bootstrapping

En la Figura 14 se presenta el modelo que muestra la significancia de estos coeficientes mediante el método de bootstrapping utilizado por el SmartPLS. 
Figura 14. Algoritmo bootstrapping para los 4 constructos del modelo TPUF

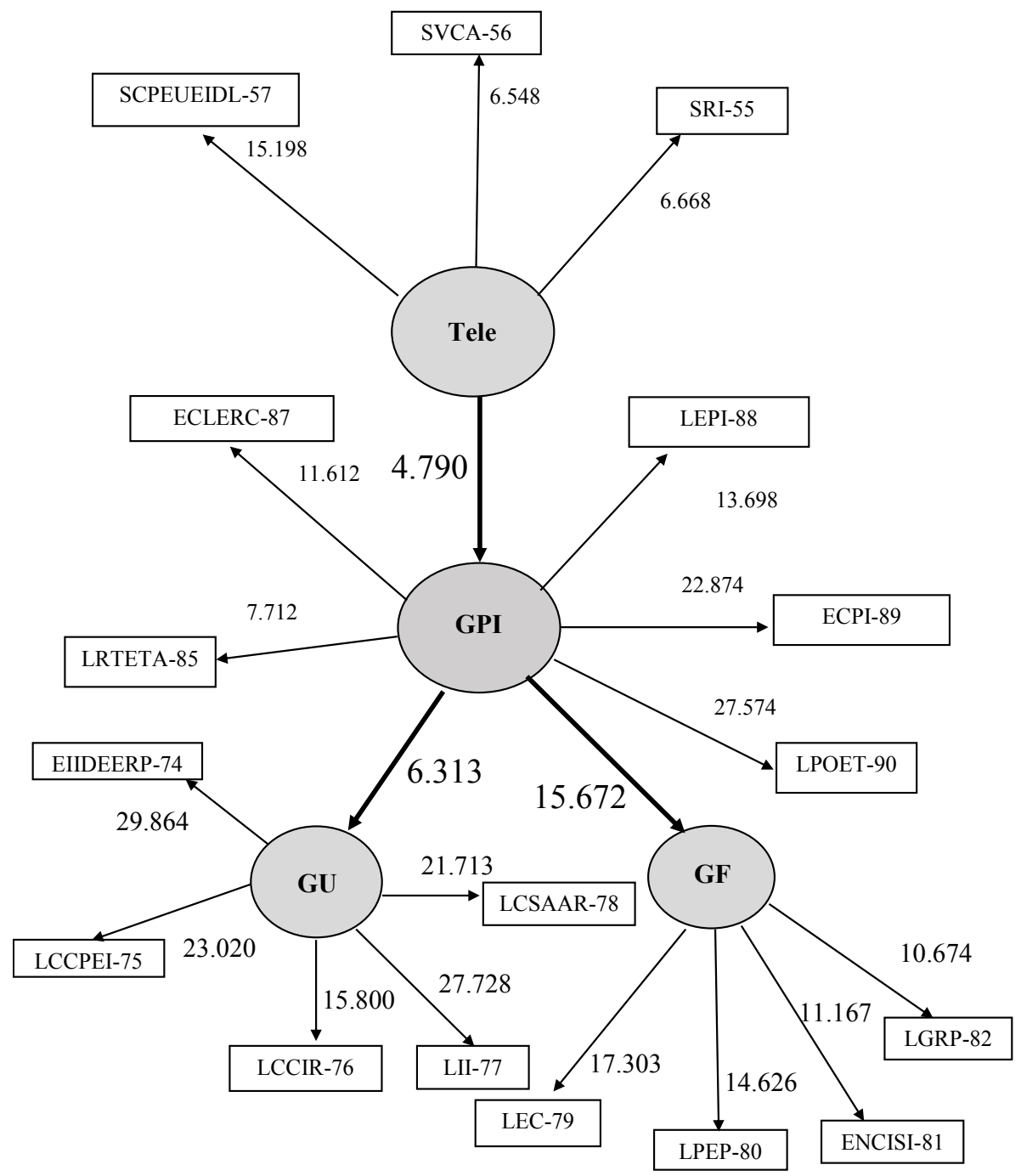

Fuente: Elaborada por el autor utilizando el software SmartPLS 
D) Evaluación del modelo.

A continuación, se muestran Tablas 7 y 8 donde se muestran los rangos de interpretación de los constructos mencionados en este modelo. Además, en la Tablas 9 y 10 se presentan la confiabilidad compuesta y el valor de impacto entre constructos del modelo TPUF.

Tabla 7. Coeficientes y su impacto en el modelo

\begin{tabular}{llll}
\hline \multicolumn{2}{l}{ Rango para coeficientes } & Valoración de impacto \\
\hline 0.00 & a & 0.09 & Imperceptible \\
0.10 & a & 0.15 & Perceptible (apenas) \\
0.16 & a & 0.19 & Considerable \\
0.20 & a & 0.29 & Importante \\
0.30 & a & 0.50 & Fuerte \\
Mayores a & 0.50 & Muy Fuerte \\
\hline
\end{tabular}

Fuente: Guía integrada por Rositas (2005).

Tabla 8. Bootstrapping y su impacto en el modelo

\begin{tabular}{lll}
\hline Rango de valoración & Significancia & P. Value \\
\hline t mayor o igual a 3.1 & $\begin{array}{l}\text { Altamente } \\
\text { significativo }\end{array}$ & 0.0001 \\
t mayor o igual a 2.33 y menor a 3.1 & $\begin{array}{l}\text { Considerablemente } \\
\text { significativo }\end{array}$ & 0.01 \\
t mayor o igual a 1.68 y menor a 2.3 & Significativo & 0.05 \\
t menor a 1.68 & No significativo & Mayor a .05 \\
\hline
\end{tabular}

Fuente: Guía integrada por Rositas (2005). 
Tabla 9. Confiabilidad compuesta del modelo TPUF

\begin{tabular}{lcccc}
\hline \multicolumn{1}{c}{ Constructo } & $\begin{array}{c}\text { Coeficiente de } \\
\text { determinación } \\
\mathbf{R}^{2}\end{array}$ & $\begin{array}{c}\text { Coeficiente } \\
\text { (Alfa de } \\
\text { Cronbach) }\end{array}$ & $\begin{array}{c}\text { Confiabilidad } \\
\text { Promedio } \\
\text { Extraída (AVE) }\end{array}$ & $\begin{array}{c}\text { Composite } \\
\text { Reliability } \\
\text { (CR) }\end{array}$ \\
\hline Telecomunicaciones & & .745 & .658 & .852 \\
Gestión de Procesos Internos & .330 & .876 & .672 & .910 \\
Gestión Usuarios & .439 & .931 & .783 & .947 \\
Gestión Financiera & .619 & .848 & .687 & .898 \\
\hline
\end{tabular}

Fuente: Tabla elaborada por el autor.

Tabla 10. Valor de impacto entre constructos modelo TPUF

\begin{tabular}{|c|c|c|c|}
\hline Relación entre Constructos & & $\begin{array}{c}\text { Coeficiente } \\
\text { o Beta }\end{array}$ & Valor del Impacto \\
\hline $\begin{array}{l}\text { Servicio de } \\
\text { Telecomunicaciones }\end{array}$ & $\begin{array}{l}\text { Gestión de } \\
\text { Procesos } \\
\text { Internos }\end{array}$ & 0.574 & Muy Fuerte \\
\hline $\begin{array}{l}\text { Gestión de Procesos } \\
\text { Internos }\end{array}$ & $\begin{array}{l}\text { Gestión } \\
\text { Usuarios }\end{array}$ & 0.663 & Muy Fuerte \\
\hline $\begin{array}{l}\text { Gestión de Procesos } \\
\text { Internos }\end{array}$ & $\begin{array}{l}\text { Gestión } \\
\text { Financiera }\end{array}$ & 0.787 & Muy Fuerte \\
\hline
\end{tabular}

Fuente: Tabla elaborada por los autores.

Tabla 11. Significancia entre constructos modelo TPUF (Algoritmo bootstrapping)

\begin{tabular}{llcc}
\hline Relación entre Constructos & \multicolumn{1}{c}{$\begin{array}{c}\text { Coeficiente } \\
\text { o Beta }\end{array}$} & Valor del Impacto \\
\hline $\begin{array}{l}\text { Servicio de } \\
\text { Telecomunicaciones }\end{array}$ & $\begin{array}{c}\text { Gestión de } \\
\text { Procesos } \\
\text { Internos }\end{array}$ & 4.785 & $\begin{array}{c}\text { Altamente } \\
\text { significativo }\end{array}$ \\
$\begin{array}{l}\text { Gestión de Procesos } \\
\text { Internos } \\
\text { Gestión de Procesos } \\
\text { Internos }\end{array}$ & $\begin{array}{l}\text { Gestión } \\
\text { Usuarios } \\
\text { Gestión } \\
\text { Financiera }\end{array}$ & 15.672 & $\begin{array}{c}\text { Altamente } \\
\text { significativo } \\
\text { Altamente } \\
\text { significativo }\end{array}$ \\
\hline
\end{tabular}

Fuente: Tabla elaborada por los autores. 


\section{Discusión de resultados}

A continuación, presentamos una discusión sobre la fiabilidad del modelo, así como los resultados sobre las hipótesis planteadas al inicio de este artículo, así como la interpretación de los mismos.

\section{Fiabilidad}

Para asegurar la validez de contenido, se definió como primer paso el identificar los ítems o reactivos que han sido utilizados en estudios de campo anteriores y que en forma satisfactoria y estadísticamente han sido significativos al representar las variables consideradas en el modelo conceptual.

Para el análisis de fiabilidad se utilizó el indicador de Alfa Chronbach que permite comprobar si cada ítem mide lo mismo y si la respuesta tiende a covariar, es decir, si los sujetos responden de una manera coherente y de esta manera se pueda deducir que los ítems se varían en el mismo sentido. Cuanto más cerca se encuentre el valor del alfa a 1 mayor es la consistencia interna de los ítems analizados. El coeficiente de Alfa Cronbach se considera aceptable cuando al menos es de 0.70 (Nunnally, 1967) y (Cronbach \& Meehl,1995).

Después de realizar la evaluación se puede determinar que el instrumento de muestreo utilizado para esta investigación es confiable ya que muestra en su mayoría niveles aceptables de Alfa Cronbach. De la tabla 9 podemos decir que los coeficientes de confiabilidad (Alfa de Cronbach) para cada criterio obtenido en esta investigación oscilaron de 0.745 y 0.931 .

Resultado de las hipótesis planteadas en el modelo

A partir de los resultados obtenidos y mostrados en la Tabla 10, todos los contructos están fuertemente relacionadas para este modelo.

La Tabla 11 muestra los resultados obtenidos al correr Bootstrapping y podemos obtener que una hipótesis tiene alta significancia y otra es considerablemente significativa en el modelo.

A continuación, se expresan las hipótesis de trabajo, planteadas estadísticamente como hipótesis nulas. 
$H_{1}$ : A mejor infraestructura en telecomunicaciones, no mejora la gestión de procesos internos.

La hipótesis 1, supone que a mejores servicios en las telecomunicaciones que se encuentran en las IES, no mejora la gestión de los procesos internos, y con los datos de las Tablas 10 y 11 los resultados muestran que existe una relación muy fuerte y altamente significativa respectivamente. Se puede concluir que la hipótesis nula $(\mathrm{H} 1)$ se rechaza; esto es, cuando se mejoran los servicios de las telecomunicaciones en las IES, se mejora la gestión de procesos.

$\mathrm{H}_{2}$ : A mejor gestión en los procesos internos, no es mejor es la gestión de usuarios.

La hipótesis 2, supone que a mejor gestión de procesos internos que se realizan en las IES, no mejora la gestión que tiene los usuarios y con los datos de las tablas 10 y 11 los resultados muestran que existe una relación muy fuerte y altamente significativa respectivamente. Se puede concluir que la hipótesis nula $(\mathrm{H} 2)$ se rechaza; esto es, cuando se mejora la gestión de los procesos internos en las IES, se mejora la gestión de usuarios.

$H_{3}$ : A mejor gestión en los procesos internos, no es mejor la gestión financiera.

La hipótesis 3, supone que a mejor gestión de procesos internos que se realizan en las IES, no mejora la gestión financiera y con los datos de las tablas 10 y 11 los resultados muestran que existe una relación muy fuerte y altamente significativa respectivamente. Se puede concluir que la hipótesis nula $(\mathrm{H} 3)$ se rechaza; esto es, cuando se mejora la gestión de los procesos internos en las IES, se mejora la gestión financiera.

Interpretación del modelo en ejecución

En la Figura 12 relacionada con la estimación del modelo en su ejecución PLS, se presenta el modelo de salida, donde se muestran los coeficientes de regresión, que por tratarse de variable estandarizadas también corresponden a coeficientes de correlación. También se presenta el coeficiente de determinación del modelo. Como puede observarse el $33 \%$ de la gestión de 
procesos internos está determinada por el servicio de las telecomunicaciones que existen en las IES ( =.57). El constructo gestión de usuarios y gestión financiera están determinados por la gestión de procesos internos en un $43.9 \%$ y $61.9 \%$, con una $=.676$ y $=.787$ respectivamente. La gestión de procesos internos tiene un impacto muy fuerte y altamente significativo. Los constructos gestión de usuarios y gestión financiera también tienen un impacto muy fuerte y una relación altamente significativa del constructo gestión de procesos internos

\section{Varianza promedio extraída}

Se examinó la Varianza Promedio Extraída (AVE) la cual estima la cantidad de la varianza latente capturada en conjunto por los indicadores. La validez convergente de los indicadores, en conjunto y en promedio para cada constructo (AVE) debe ser mayor al 0.50; esto significa que en cada constructo se captura más del $50 \%$ de su varianza, excediendo por lo tanto al porcentaje no explicado o capturado (Chin \& Newsted, 1999).

Para medir la confiabilidad y la consistencia de las variables latentes estimadas se utilizó la confiabilidad compuesta (Composite Reability, CR) la cual mide la consistencia interna de los reactivos que miden los constructos. Los valores menores a .70 indican que los reactivos pueden no estar relacionados o pueden medir más de un constructo (Chin, 1998).

En la tabla 9 podemos observar que En este modelo los constructos varían entre .852 y .947 , lo cual nos indica que existe una buena consistencia interna entre los reactivos y la varianza promedio extraída que oscila entre 0.658 y 0.783

\section{Conclusiones y recomendaciones}

Los retos de un mundo globalizado exigen a las instituciones de educación superior pública en México que se invierta en forma significativa en la infraestructura de las telecomunicaciones. Los procesos internos cada vez requieren más eficiencia y eficacia para cubrir las necesidades institucionales. Las IES se tienen que adaptar a los cambios del siglo XXI.

En los resultados anteriores podemos observar que existen muchas variables que están relacionadas a los servicios de telecomunicaciones, 
gestión de procesos internos, gestión que realizan los usuarios de las mismas, así como la gestión financiera institucional.

En cuanto a los resultados estadísticos del modelo planteado, estos nos indican que todos los constructos tienen una relación muy fuerte y son altamente significativos para las IES.

Por tal motivo se rechazan las 3 hipótesis propuestas en esta investigación; sin embargo, se buscará analizar otros elementos con mayor accesibilidad en la obtención de sus datos que permita ampliar esta investigación y determinar las variables que impacten la GPI dentro de la institución educativa.

El estudio demuestra que se tiene que tomar en cuenta más variables independientes adicionales. En la medida que las instituciones incrementen los servicios de telecomunicaciones se obtendrán mayores beneficios institucionales

Las instituciones educativas de nivel superior tienen que generar estrategias que coadyuven a los diversos servicios, también se tiene que romper paradigmas de actitud, cambiar formas de relación laboral y social, Además, se tienen que realizar cambios del entorno para evitar la monotonía laboral entre otros aspectos importantes.

A través de este estudio hemos podido validar algunos estudios anteriores y nuestra contribución principal es proporcionar a los investigadores información que demuestra estadísticamente el impacto de las telecomunicaciones en las gestiones de usuarios y financieras en las IES públicas de México.

De los resultados mostrados y del análisis de los mismos, de la investigación: Impacto de las Telecomunicaciones en la gestión de las instituciones de educación superior públicas de México: Un Modelo de Ecuaciones Estructurales se puede extraer que, la transformación explicada por el proceso de la democratización de la educación, produjo en los últimos años importantes cambios en el contexto universitario apoyado esto en palomares (2008). También destaca que se confirma lo dicho por la OCDE (1999) que la aparición de un nuevo modelo de universidad que adopta de forma creciente la llamada "tercera misión" tiene un surgimiento de la sociedad del conocimiento, el fenómeno de la mundialización de los servicios, entre otros temas. 
Finalmente, sostenemos que existe la necesidad de realizar más estudios regionales en distintos ámbitos sociales, y los resultados parciales obtenidos sean como fundamento para una investigación de mayor alcance, donde el instrumento considere otras variables para un análisis más profundo.

La definición de proyectos tendientes a incrementar el conocimiento técnico-científico requiere hacerse con base en una visión integral que contemple todos los aspectos sociales y económicos del país dentro del marco de prioridades de desarrollo nacional

Se requiere de la elaboración de estrategias regionales y nacionales que permitan determinar cuándo, cómo, dónde y a quién se debe asignar diversos tipos de recursos que permitan incrementar los servicios y gestiones institucionales

\section{Limitaciones y futuras líneas de investigación}

Este trabajo utiliza información de corte transversal. Sin embargo, futuras investigaciones podrían analizar las relaciones dinámicas de las variables, a través de información longitudinal, con una muestra más amplia superando las restricciones presupuestarias y logísticas que dicha iniciativa implica. El trabajo se enfocó a analizar la realidad actual, por lo que futuros esquemas en las IES de México cambiarán las competencias de la sociedad en general.

También, próximos estudios podrían analizar el impacto de las telecomunicaciones en las gestiones de los usuarios y las financieras mediante modelos más integrales, donde se puedan incluir otros aspectos que puedan influir significativamente. Sería interesante estudiar los valores y la cultura poblacional y relacionarlos a los servicios y gestiones de las IES de México.

\section{Referencias}

Aja, L. (2002). Gestión de información, gestión del conocimiento y gestión de la calidad en las organizaciones, ACIMED, 10(5), 7-8.

Bricall, J. (2000). Universidad 2mil. Madrid: CRUE.

Bueno, E. (2007). La tercera misión de la universidad. El reto de la transferencia del conocimiento, Revista Madrid, 41, marzo-abril. 
Cave, M., Hanney, S., Henkel, M. \& Kogan, M. (1988, 1997). The use of performance indicators in higher education (1sted. \& 3rded.). London and Bristol: Jessica Kingsley Publishers.

Chin, W. W. (1998). Issues and opinion on structural equation modeling, MIS Quarterly, 22(1), 7-16.

Chin, W. W. \& Newsted, P. R. (1999). Structural equation modeling analysis with small samples using partial least squares. Statistical strategies for small sample research, 1(1), 307-341.

Clark, B. (1997). Creando universidades emprendedoras en Europa, Revista Valenciana d'Estudis Autonomics, 21, 373-392.

Clark, B. (1998). The entrepreneurial university demand and response, Tertiary Education Management, 4(1), 5-15.

Commission of the European Communities (2006). Communication from the Commission Delivering on the modernization agenda for universities: education, research and innovation. Brussels: Commission of the European Communities.

Consejo de Universidades (1999). Indicadores en la Universidad: Información y decisiones. Madrid: MEC

Córdoba, F. G. (2002). El cuestionario: recomendaciones metodológicas para el diseño de cuestionarios. Editorial Limusa.

Cronbach, L. J. \& Meehl, 0. E. (1995). Construct validity in psychological tests. Psychological Bulletin, 281-302.

Cuenin, S. (1986). International Study of the Development of Performance Indicator in Higher Education. Paper given to OECD, IMHE Project, Special Topic Workshop.

Davies, J. (2001). The emergence of entrepreneurial cultures in European universities. Higher Education Management, 13(2), 25-43.

Declaración de Principios. Cumbre Mundial sobre la Sociedad de la Información. Ginebra, Suiza, del 10 al 12 de diciembre de 2003. (2003) [en línea] [fecha de acceso 10 de noviembre 2016] Disponible en URL: https://www.itu.int/dms_pub/itus/md/03/wsis/doc/S03-WSIS-DOC-0004!!PDF-S.pdf

Díaz, M. D. M. (1999). La evaluación de la enseñanza. Propuesta de indicadores para las titulaciones. En: Consejo de Universidades (editor), Indicadores en la Universidad: Información y decisiones. Madrid: MEC, 413-430.

Garcia, A., Gutiérrez, A. \& Pérez, M. (2006). Analysis of the evaluation process of the research performance: an empirical case, Scientometrics, 67(2), 213-230.

García, N. (1997). Análisis de la decisión individual de uso permanente en el proceso de asimilación de nuevas tecnologías. Tesis para obtener el grado de Doctor en Ingeniería. San Nicolás de los Garza: Universidad Autónoma de Nuevo León, Facultad de Ingeniería Mecánica y Eléctrica.

Garza J., Lerma, C. \& Terriquez, O. (2014). Modelo Baldrige en una empresa manufacturera y su modelación de ecuaciones estructurales con mínimos cuadrados parciales. Caso de estudio, Innovaciones de Negocios, 11(22), 329-359. 
Gulbrandsen, M. \& Slipersaeter, S. (2007). The third mission and the entrepreneurial university model. In: Bonaccorsi, A. \& Daraio. C. (eds.), Universities and Strategic Knowledge Creation. Cheltenham, UK/ Northampton, MA: Edward Elgar, 112-143.

Keller, G. (1999). The new importance of strategic management at universities, International Seminar on Strategic Management and Quality of Universities. Barcelona: Universidad Politécnica de Cataluña.

Kerlinger. F. \& Lee H. (2002). Investigación del comportamiento: Métodos de investigación del comportamiento: Métodos de investigación en ciencias sociales. México. Mc Graw-Hill Interamericana Editores. $4^{a}$ Edición.

Kvilon, K. (2004). Las tecnologías de la información y la comunicación en la formación docente. Francia: UNESCO.

Lastres Segret, J. A.; Moreno Sanabria, R. C. (2002). La influencia de las tecnologías digitales sobre la e-economía. Comunicación presentada a la III Bienal Iberoamericana de la Comunicación. Universidad de Las Américas-Puebla, Cholula, México, diciembre.

Lou (2001). BOE n. 307 de 24/12/2001.

Lou (2007). BOE n. 89 de 13/04/2007.

Macau, R. (2004). TIC: ¿Para qué? Funciones de las tecnologías de la información y la comunicación en las organizaciones. RUSC. Universities and Knowledge Society Journal, 1(1), 1-12.

Molas, J. (2002). Measuring third stream activities. Final report to the Russell Group of Universities, Brighton: University of Sussex, Science and Technology Policy Research.

Mora, J. (1991). Evaluación y rendimiento institucional. Quaderns de Treball, 199, Universitat de València, Facultat de Ciències Econòmiques i Empresarials.

Moscoso, S., Perez, J. \& Holgado, F. (1999). Indicadores de calidad en la universidad de Sevilla. Análisis de contenido. En: Consejo de Universidades (editor), Indicadores en la Universidad: Información y decisiones. Madrid: MEC, 385-400.

Nunnally, J. C. (1967). Psychometric Theory, 1st ed., New York: McGraw-Hill.

OCDE (1999). University research in transition. Paris: OCDE.

OCDE (2004). Education at a Glance. OCDE Indicators. Paris: OCDE.

Palomares, D., García A., Castro, E. (2008). Evaluación de las instituciones de educación superior: revisión bibliográfica de sistema de indicadores, Revista Española de Documentación Científica, 31(2), 205-229.

Pla, A. \& Villarreal, E. (2001). Measuring the internal efficiency of universities: Social demand, diversity and rising costs. Paper presented at the XXIII Annual EAIR Forum. Porto: Univesity of Porto.

UIT (2000), Libro Azul: Políticas de Telecomunicaciones para las Américas, Ginebra: Unión Internacional de Telecomunicaciones.

Rositas J. (2014). Los tamaños de las muestras en encuestas de las ciencias sociales y su repercusión en la generación del conocimiento, Innovaciones de Negocios, 11(22) 235-268

Serrano, A. (2000). Las telecomunicaciones en Latinoamérica. Retos y perspectivas. Cd. México: Pearson Educación. 
Villarreal, E. (1999). La utilización de indicadores de rendimiento en la financiación de la educación superior. En: Consejo de Universidades (editor). Indicadores en la Universidad: Información y decisiones. Madrid: MEC, 65-80. 